\title{
Platelet count as a prognostic indicator in pregnancy induced hypertension
}

\author{
Tejeswini K. K. ${ }^{1}$, Anitha G. S. ${ }^{1}$, Nandagopal K. M. ${ }^{2}$
}

\begin{abstract}
${ }^{1}$ Department of Obstetrics and Gynecology, Bangalore Medical College and Research Institute, Bangalore, India
${ }^{2}$ Department of Obstetrics and Gynecology, Sri Siddhartha Medical College, Tumkur, India
\end{abstract}

Received: 23 November 2015

Revised: 26 February 2016

Accepted: 01 March 2016

\section{*Correspondence:}

Dr. Tejeswini K. K.,

E-mail: drtejeswini11@gmail.com

Copyright: () the author(s), publisher and licensee Medip Academy. This is an open-access article distributed under the terms of the Creative Commons Attribution Non-Commercial License, which permits unrestricted non-commercial use, distribution, and reproduction in any medium, provided the original work is properly cited.

\begin{abstract}
Background: Hypertensive disorders are most common medical complications of pregnancy, and are one of the major causes of maternal and fetal morbidity and mortality. Thrombocytopenia complicating hypertensive disorders of pregnancy are responsible for approximately $20 \%$ of all cases of thrombocytopenia during pregnancy.

Our study was done to assess the utility of platelet count as a prognostic indicator in pregnancy induced hypertension to recognize and manage early the complications arising and to have a better pregnancy outcome.

Methods: This study includes 76 cases of pregnancy induced hypertension over a period of 18 months. Platelet estimation was done for all cases and patients with documented platelet count of less than 1,50,000/cumm was documented as thrombocytopenia.

Results: Of the 76 cases of pregnancy induced hypertension, 32 (42.1\%) were diagnosed with thrombocytopenia, and an increased incidence of maternal and fetal morbidity \& mortality was observed.

Conclusions: Our study and the results show that the assay of platelets can be considered as one of the prognostic tool in management of hypertensive disorders of pregnancy.
\end{abstract}

Keywords: Pregnancy induced hypertension, Thrombocytopenia

\section{INTRODUCTION}

Thrombocytopenia is an associated phenomenon of $\begin{array}{lll}\text { Pregnancy induced hypertension (PIH). } & 1\end{array}$ Thrombocytopenia complicates $7-8 \%$ of all pregnancies. This increasing incidence of the disease is mainly attributed to automated complete blood count analyser which routinely includes platelet count. In pregnancy, most of this decline occurs in third trimester and is associated with a shift in the histogram of platelet count distribution.

Thrombocytopenia is defined as subnormal number of platelets in the circulating blood. ${ }^{2}$ In pregnancy, it results from a variety of causes ranging from benign disorders such as gestational thrombocytopenia to severe complicated life threatening conditions such as HELLP syndrome. Thrombocytopenia resulting from pregnancy induced hypertension is responsible for approximately $20 \%$ of all cases of thrombocytopenia during pregnancy.

In pre eclampsia, usually the thrombocytopenia is mild to moderate but patients with eclampsia can develop severe thrombocytopenia and are more likely to have HELLP syndrome, which is a subset of pre eclampsia. Thrombocytopenia is a key and necessary component of this syndrome.

The mechanism of development of thrombocytopenia is mainly from 4 processes - artifactual thrombocytopenia, 
deficient platelet production, accelerated destruction and pooling of platelets. Thrombocytopenia is mainly characterized by bleeding from small vessels. The time of onset of these disorders during pregnancy and their associated clinical manifestations often overlap and thereby makes the diagnosis of specific disorders difficult.

Thrombocytopenia carries a risk for both the mother and her fetus, associated with substantial maternal or neonatal morbidity \& mortality. Therefore it is of utmost importance to institute specific therapies promptly and improve the maternal and neonatal outcome. Hence this study was done to know the incidence of thrombocytopenia in pregnancy induced hypertension and its effect on maternal and fetal outcome.

\section{Aims and objectives}

Maternal and fetal outcome is adversely affected by abnormalities of platelets in pregnant women resulting from number of diverse etiologies. However, early diagnosis of thrombocytopenia in pregnancy induced hypertension and immediate intervention can considerably improve the maternal and fetal outcome.

Hence this study has been taken to know the utility of platelet count as a prognostic indicator in Pregnancy Induced Hypertension and thereby aid in early diagnosis and management improving maternal \& fetal outcome.

\section{Platelet structural and functional anatomy}

\section{Light microscopy}

On Wright-giemsa stained blood smear, platelet appears as small, enucleate ovoid or round cells with a pale grayish-blue cytoplasm that contains homogeneously distributed purple- red granule.

\section{Platelet physiology}

\section{Platelet adhesion}

Vascular endothelium actively inhibits platelet activation by synthesis and secretion of prostacyclin and nitric oxide with vascular injury, components of sub endothelium including collagen \& microfibrils, promote platelet adhesion by inducing VWF binding to platelet gplb. VWF is present in the subendothelial matrix as well as in blood plasma and also is secreted by platelets after activation. The initial gpIb/VWF mediated platelet contact with subendothelium is reinforced by the platelet gp IIb-IIIa complex binding VWF and other adhesive ligands to firmly attach a layer of platelets to the damaged vascular surface. ${ }^{3}$

\section{Platelet activation \& aggregation}

If an activating stimulus is sufficient (threshold level) platelet activation occurs. This is associated with granule secretion (the release reaction) and stimulation of prostaglandin synthesis. Granule contents are released through canalicular system that connects the interior of the platelet with the external environment. Prostaglandin synthesis is initiated. When phospholipase A2 generates arachidonic acid from platelet phospholipids arachidonic acid subsequently is converted by platelet cyclooxygenase to labile endoperoxides (PGG2, PGH2) that then are converted by thromboxane synthatase to TX A2, a potent activator and vasoconstrictor. Platelet activation also leads to expression of the GP IIb- IIIa receptor fibrinogen binding and platelet aggregation.

\section{Laboratory evaluation of platelet number \& function}

In patients suspected of a disorder of haemostasis, defects in platelet number or function, impaired coagulation or abnormalities in vascular function should be considered.

\section{Platelet count}

This screening test is performed routinely as a part of the complete blood count using automated particle counters. A typical reference range is $1,50,000-4,40,000 / \mathrm{mc}^{4}$.

When thrombocytopenia is reported confirmation should be obtained by evaluation of the patients peripheral smear. Approximately $1 \%$ of patients has artificial thrombocytopenia due to EDTA- induced platelet clumping and do not have true thrombocytopenia. The platelet count is a valuable rapid screening test in assessing acute obstetric haemostatic failure, particularly in helping the attendants together with other assessments to diagnose the presence and severity of disease.

\section{Bleeding time}

The length of time a small skin wound continues to bleed depends largely on the number and function of platelets, i.e their ability to form plugs at the site of injury (normal range upto 7-10 minutes). It is only rapidly performed invivo test of platelet vascular interaction. The bleeding time cannot predict bleeding, blood loss or transfusion requirements.

\section{Antiplatelet antibody tests}

Antiplatelet antibody test may be a greater clinical utility in the evaluation of neonatal alloimmune thrombocytopenia. Enzyme linked immunosorbent assays (ELISA) are available to diagnose platelet alloantibodies. 


\section{Thrombopoietin (TPO) assay}

Very low TPO levels are associated with peripheral platelet destruction, including ITP, where an elevated TPO levels are seen in marrow failure. ${ }^{6}$

If there results are confirmed in additional studies, a low TPO level might replace the necessity for bone marrow examination in certain patients with thrombocytopenia.

\section{Activated partial thromboplastin time}

This test is also known as the partial thromboplastin time which kaolin and the kaolin cephalin clotting time. It gives a crude assessment of the integrity of the intrinsic coagulation system. The normal range usually lies between 35 and 45 seconds, but all tests must always be compared with known normal plasma. Prolongation occurs with isolated factor deficiencies of factors VIII, IX and XI and also in the presence of therapeutic heparin levels.

\section{Prothrombin time}

This test measure the clotting time of citrated plasma after the addition of an optimal concentration of tissue extract (thromboplastin). It is a measure of the overall efficiency of the extrinsic clotting system. Normal range is approximately 10-14 seconds. It is prolonged in isolated factor VII deficiency and in liver failure, and it is the test used to monitor dosage of warfarin (INR) in those on oral anticoagulants.

\section{Thrombin time}

This test is a measure of the final common pathway of the extrinsic and intrinsic coagulation systems. Normal plasma will give a thrombin clotting time of 15-19 seconds. The commonest causes of a delayed TT are the presence of heparin. Both the clotting time and the appearance of the clot are informative.

\section{Fibrinogen estimation}

It should be remembered that in late pregnancy. I.e. after 30 week's gestation, the normal non pregnant range of fibrinogen, $20 .-4.5 \mathrm{~g} / 1$ is increased to $4.0-6.0 \mathrm{~g} / 1$. Reduction of fibrinogen is seen in severe consumptive coagulopathy and in rare inherited deficiencies of fibrinogen.

\section{Detection of fibrinogen/fibrin degradation products}

Healthy subjects have FDP concentration of less than $10 \mathrm{mg} / \mathrm{ml}$. concentrations between 10 and $40 \mathrm{mg} / \mathrm{l}$ are seen in acute inflammatory disorders-in acute venous and arterial thrombosis and after strenuous exercise or major surgery. High levels of FDPs are seen in association with severe acute disseminated intravascular coagulation and following thrombolytic therapy with streptokinase.

\section{Pathophysiology of thrombocytopenia}

It is the most common cause of abnormal bleeding. The pathophysiology of thrombocytopenia is similar to that of anemia. This, despite the number and diversity of disorders that may be associated etiologically, thrombocytopenia results from only four processes

1. Artifactual thrombocytopenia ${ }^{7}$

2. Deficient platelet production

3. Accelerated platelet destruction, and

4. Abnormal distribution or pooling of the platelets within the body.

\section{Platelets in pregnancy induced hypertension}

Platelets play a crucial role in the pathophysiology of preeclampsia by promoting vascular damage and obstruction, leading to tissue ischaemia and further damage. ${ }^{9}$ Thromboxane A2 the major product of arachidonic acid metabolism in platelets, is a potent vasoconstrictor and platelet- aggregating agent. As it has a short half-life it is normally measured as its stable hydration product, thromboxane B2. The effects of thromboxane A2 are normally counterbalanced by prostacyclin, a potent vasodilator and anti-platelet prostanoid which is the major product of arachidonic acid metabolism in vascular endothelium and which plays an important role in protecting the endothelium and limiting damage by inhibiting platelet aggregation and promoting vasodilatation. These two substances function as local hormones and are thought to be important in the control of the platelet-endothelium interaction. They oppose each other though the regulation of platelet adenylate cylase, which controls cAMP production and thereby platelet free calcium concentration; this links receptor occupancy with cellular response. Pro-aggregatory substances such as thromboxane A2 inhibit adenylate cyclase, allowing free intracellular cAMP, reducing free intracellular calcium and inhibiting platelet activation.

The platelet specific protein B- thromboglobulin marker of platelet activation in vivo has been found to be increased in pregnancy induced hypertension. This correlates with proteinuria and serum creatinine, and suggests a link between platelet activation with renal microvascular damage. ${ }^{10}$

The platelet content of 5-hydroxytryptamine (5-HT) is reduced in pre-eclampsia, indicating platelet aggregation and stimulation of the platelet release reaction in vivo. Increased platelet thromboxane $\mathrm{A} 2$ production in vivo has been shown to occur in pre-eclampsia, complicated by intrauterine growth retardation. Gowland et al found reduced platelets reactivity in whole blood in women with pre-eclampsia compared to normal controls, although there was no difference in thromboxane A2 production in vivo. The report of Gowland et al would be in keeping differences in patient severity, as platelet reactivity may vary according to the stage of the disease 
process, with increased reactivity perhaps occurring in the early stages of the disease and platelet exhaustion in advanced disease. $^{11}$

The damage in the coagulation system and in platelet function support the concept that disseminated intravascular coagulation occurs in patients with pregnancy induced hypertension. A 'coagulation index' of serum fibrinogen degradation product, platelet count and plasma factor VIII has been shown to correlate with a 'clinical index' of disease severity, highlighting the association of the two conditions.

\section{Gestational thrombocytopenia}

This condition has also been termed benign or incidental thrombocytopenia. There is no history of autoimmune disease and the other possible causes of thrombocytopenia such as pre eclampsia have been excluded. Typically, the condition is mild with a platelet count of 120-150 x 10/1, although moderate (50-120 x $10 / 1)$ and severe $(<50 \times 10 / 1)$ thrombocytopenia have been described ${ }^{12}$. Many studies have documented the normal fetal and maternal outcome in such cases. Thus, some degree of gestational thrombocytopenia is considered normal. Burrows and Kelton reported that 6.6 percent of 15,471 pregnant women had platelet counts $<150,000 / \mathrm{L}$, and in 1.2 percent, they were $<100,000 / \mathrm{L}$. They reported that almost 75 percent of 1027 women whose platelet counts were $<150,000 / \mathrm{L}$ was found to have normal-variant incidental thrombocytopenia. Of the remainder, 21 percent had a hypertensive disorder of pregnancy, and 4 percent had an immunological disorder. $^{13}$

In the largest study involving over 15,000 women, Burrows and Kelton noted the incidence of thrombocytopenia to be $4.8 \%$ having excluded autoimmune disease and pre- eclampsia. ${ }^{14}$ There was no neonatal morbidity. The mechanism of neonatal thrombocytopenia has not been determined and it has recently been suggested that in nearly $50 \%$ of such cases there may be an underlying maternal autoimmune condition. $^{15}$

The diagnosis of gestational thrombocytopenia relies on the exclusion of the other possible causes, and is helped by the knowledge that a platelet count was normal either before, or in the early part of pregnancy. If this is the case, then prognosis appears to be extremely good and the platelet counts tend to return to a normal value following delivery. Aggressive medical or obstetric intervention should be avoided. Delivery should be attempted vaginally unless there are other obstetric indications for operative delivery. Postnatally, fetal cord blood should be sampled at the time of delivery and serial maternal counts undertaken to ensure that the maternal count returns to a normal value.

\section{HELLP syndrome}

A serious variant of pre-eclampsia is HELLP syndrome. First coined by Weinstein in 1982, HELLP is an acronym for hemolysis, elevated liver function, and low platelet count. Of the array of microangiopathies that may occur during pregnancy, the most frequently encountered disorder of microangiopathic red cell and platelet destruction is HELLP syndrome.

\section{Diagnosis}

\section{Hemolysis}

Abnormal peripheral smear

Total bilirubin $>1.2 \mathrm{mg} / \mathrm{dl}$

Lactate dehydrogenase $>600 \mathrm{U} / \mathrm{L}$

Elevated liver enzymes

Aspartate aminotransferase $>70 \mathrm{U} / \mathrm{L}$

Lactic dehydrogenase $>600 \mathrm{U} / \mathrm{L}$

\section{Low platelet count}

\section{Platelets $<100,000 / \mathrm{mm}$}

Martin et al at the University of Mississippi have further categorized HELLP syndrome into different classes based on the severity of the thrombocytopenia.

Class 1 Platelets $\leq 50,000 / \mathrm{mm}$

Class 2 Platelets $>50,000 / \mathrm{mm}$ to $\leq 100,000 / \mathrm{mm}$

Class 3 Platelets $>100,000 / \mathrm{mm}$

\section{Etiology and pathophysiology of disease}

As with other microangiopathies, a major component of the underlying perturbation of HELLP syndrome involves endothelial injury. Intimal injury stimulates fibrin deposition in the vessel lumen with subsequent disruption of the formed elements flowing in contact with the area of injury. Activated platelets release vasoconstrictive substances, including serotonin and thromboxane A. Platelet aggregation causes further endothelial injury impairing prostacylin production. Reflecting endothelial damage in the pathogenesis of HELLP syndrome, endothelin, a potent vasoconstrictor, is found in higher levels in pre-eclamptic patients than in controls. ${ }^{17}$

Fibrin obstructs hepatic sinusoids and this obstruction produces hepatocellular injury and pain. Hyaline material is deposited in the periportal or parenchymal areas along with hemorrhage. Large subcapsular or intraparenchymal hemorrhage may be seen periodically. Fatty infiltration has been reported by Barton et al in 4 of 11 patients with HELLP syndrome undergoing liver biopsy at the time of cesarean delivery. ${ }^{18}$ Unlike acute fatty liver of pregnancy, 
where vacuolization is seen in the central zone, with HELLP syndrome, it is seen in the periportal region.

\section{Presentation}

Patients at risk for HELLP syndrome tend to be older (mean age 25 years) and multiparous when compared with patients developing severe pre-eclampsia/ eclampsia (mean age, 19 years). Patients may have symptoms at any time during the second or third trimesters, but they are typically preterm. Fifteen percent of HELLP syndrome occurs in the midtrimester; $8 \%$ between 17 and 20 weeks and $7 \%$ between 20 and 26 weeks gestation. Eighteen percent of patients will have symptoms at term. Delivery is not uniformly curative because up to $30 \%$ of patients develop manifestations within 2 days after delivery (range, a few hours to 6 days postpartum). Seventy-nine percent of those patients developing HELLP syndrome in the postpartum period have an antepartum diagnosis of pre-eclampsia.

The presentation of patients with HELLP is variable. Symptoms can range from flu like illness to convulsions and gastrointestinal hemorrhage. Ninety percent of patients experience malaise, fatigue, and nonspecific complaints for a few days before seeking medical evaluation. Half of all patients report nausea and vomiting and or headache, whereas the ominous symptom of epigastric or right upper quadrant pain is present in half to two thirds of patients. With hepatic hemorrhage, the patient may complain of neck and shoulder pain in addition to the aforementioned abdominal complaints.

\section{Complications}

Obstetric complications typically seen are acute renal failure, abruption, disseminated intravascular coagulation, and sepsis. Approximately half of all patients diagnosed with HELLP syndrome do not manifest the complete laboratory picture. Cases of patients with HELLP syndrome, where at least one of the criteria for HELLP syndrome is met, tend to have relatively less maternal morbidity. ${ }^{19}$

\section{Perinatal outcome}

As expected, HELLP syndrome produces an adverse effect on perinatal mortality. Most perinatal deaths are from abruption, asphyxia, and extreme prematurity. When compared with preterm infants from pregnancies complicated by pre-eclampsia or normotensive controls, preterm infants delivered before 32 weeks gestation from mothers with HELLP syndrome had higher rates of grade 3 and grade 4 intraventricular hemorrhage as well as respiratory distress syndrome.

\section{Treatment}

Preterm patients with HELLP syndrome should be stabilized hemodynamically and transferred to a tertiary care center. Patients can be treated at a local hospital at term depending on the availability of obstetric neonatal, and blood banking services. Magnesium sulfate is administered for seizure prophylaxis regardless of blood pressure. Blood pressure, when elevated (e.g., $\geq 160 / 105$ ) is controlled with agents such as labetalol or hydralazine. Careful attention needs to be given to intravenous fluid administration as well as urinary output. ${ }^{20}$

If the pregnancy is $\geq 32$ weeks gestation, antenatal corticosteriods are administered to enhance fetal lung maturity. During the interval between steroid administration and delivery, the patient requires careful assessment for fluid overload, worsening hypertension, or coagulopathy. Most conservatively treated patients with HELLP syndrome require delivery between 1-10 days after admission. Severe persistent right upper quadrant or epigastric pain may indicate a liver hematoma with the potential for rupture and possible subsequent vascular collapse. These symptoms are indications for prompt delivery. Further, patients with abdominal pain coupled with neck or shoulder pain or hypotension or thrombocytopenia $(<20,000 / \mathrm{ml})$ will benefit from hepatic imaging.

HELLP syndrome by itself does not require cesarean delivery, and labour may be induced, if the patient is beyond 30-32 weeks gestation. Before 30 weeks gestation, a prolonged labor can be anticipated, and cesarean delivery may be useful if the patient has an unfavourable cervix. Consideration should be given to maintaining the platelet count $>20,000 / \mathrm{mm}$ if a vaginal delivery is planned. If a cesarean delivery is anticipated and the platelet count is $<50,000 / \mathrm{mm}, 6-10$ packs or units of platelets are given at the time of the skin incision, and if needed, and additional six units may be administered during uterine closure if the tissue oozes. In patients with HELLP syndrome, wound hematomas are common. Placement of an intraperitoneal drain has the additional advantage of revealing intraperitoneal hemorrhage from either the operative filed or the liver after the abdomen is closed.

\section{Postpartum}

The patient requires intensive observation for 48 hours after delivery. Hematologic parameters should begin to recover by this time. The obstetrician should expect massive shifts in fluid that has accumulated in the interstitum during the pregnancy. The patient should be carefully monitored for pulmonary edema, hepatic dysfunction and renal compromise.

Improvement in laboratory parameters, increase in urine output, and decreased mean arterial blood pressure have 
been reported by Magann, et al after the administration of corticosteroids immediately postpartum. ${ }^{21}$

\section{Thrombocytopenia in pregnancy induced hypertension}

The second most common cause of thrombocytopenia in pregnancy is thrombocytopenia complicating hypertension. Thrombocytopenia due to hypertensive disorders of pregnancy is responsible for $21 \%$ of thrombocytopenic mothers which represents an incidence of 13-15 per 1000 live births (approximately 1-2\% of all pregnancies). Thrombocytopenia by itself has long been associated with pre-eclampsia, with approximately $50 \%$ of pre-eclampsia manifesting thrombocytopenia. Typically, the thrombocytopenia in a pre-eclamptic patient is mild to moderate, with platelet counts in the range of $50,000-100,000 / \mathrm{ml}$. The diagnosis of preeclamsia is usually clinically apparent, with the ultimate treatment being delivery. Typically, the platelet count returns to normal within days after delivery, although occasionally we have seen patients who had severe and persistent thrombocytopenia that lasted for weeks after the delivery.

\section{METHODS}

\section{Sources of data}

All pregnant women admitted in the department of obstertrics and gynaecology, Sri Siddhartha Medical College from Nov 2009 to April 2012.

\section{Method of collection of data}

1. From all pregnant women with pregnancy induced hypertension admitted in to the department of obstetrics and gynaecology, SSMC.

2. Details will be entered in the proforma regarding the detailed history, period of gestation, high risk factors, complications-during present and past pregnancy, like pregnancy induced hypertension, diabetes mellitus, APLA, intra uterine death, abruption, hepatitis and Past history of pregnancy induced hypertension, hypertension, diabetes mellitus \& haemorrhagic disorders.

3. All patients with diagnosed hypertensive disorders of pregnancy with documented platelet count of $<1,50,000 /$ cumm have been considered in the group with thrombocytopenia.

\section{Inclusion criteria}

1. All pregnant women with pregnancy induced hypertension.

2. Gestational age above 20 weeks.

\section{Exclusion criteria}

1. Established coagulation and hematological disorders.

2. Gestational age below 20 weeks or chronic hypertension.

3. Preexisting renal or vascular diseases, seizure disorders, severe anaemia, liver diseases.
In our prospective study of one and a half year duration, there were 76 cases of pregnancy induced hypertension. Thrombocytopenia was one of the commonly associated abnormalities of pregnancy induced hypertension of any severity. The other causative factors include abruption, I.U.F.D and ITP, which constitute small percentage. Hence it is advisable to screen for thrombocytopenia in all cases of pregnancy induced hypertension irrespective of period of gestation and severity.

\section{RESULTS}

Table 1: Age distribution of pregnancy induced hypertension with and without thrombocytopenia.

\begin{tabular}{|llll|}
\hline \multirow{2}{*}{ Age } & \multicolumn{2}{l}{ Thrombocytopenia } & Total \\
\hline$<20$ years & With & Without & \\
\hline 21-25 years & $15(34.3)$ & $11(25)$ & $22(29 \%)$ \\
\hline 26-30 years & $3(9.3)$ & $22(48.4)$ & $37(49 \%)$ \\
\hline$>31$ years & $3(9.3)$ & $2(4.4)$ & $5(7 \%)$ \\
\hline Total & $32(100 \%)$ & $44(100 \%)$ & $76(100 \%)$ \\
\hline
\end{tabular}

Figures in parenthesis indicate percentage

The commonest age group in which thrombocytopenia seen was between 21 to 25 years. Table 1 shows that age was an important criterion and there was difference in the occurrence of thrombocytopenia in different age groups $(\mathrm{P}<0.002)$.

Table 2: Gravidity index of patients with pregnancy induced hypertension with and without thrombocytopenia.

\begin{tabular}{|llll|}
\hline \multirow{2}{*}{ Gravida } & \multicolumn{2}{l}{ Thrombocytopenia } & \multirow{2}{*}{ Total } \\
\hline Primigravida & With & Without & \\
\hline Multigravida & $14(43)$ & $21(48)$ & $38(50 \%)$ \\
\hline Postnatal & $1(3)$ & $22(50)$ & $56(47 \%)$ \\
\hline Total & $32(100 \%)$ & $44(100 \%)$ & $76(100 \%)$ \\
\hline
\end{tabular}

Figures in parenthesis indicate percentage

The association of pregnancy induced hypertension with thrombocytopenia was $32(42 \%)$. There is no statistically significant association between gravidity index and thrombocytopenia $(\mathrm{P}>0.05)$.

Table 3: Analysis of severity of pregnancy induced hypertension with and without thrombocytopenia.

\begin{tabular}{|llll|}
\multicolumn{1}{c}{ Whrombocytopenia } & Total \\
\cline { 2 - 4 } & With & Without & \\
\hline Pre eclampsia & $8(25)$ & $15(34)$ & $23(30 \%)$ \\
\hline Eclampsia & $8(25)$ & $19(43)$ & $35(46 \%)$ \\
\hline Total & $32(100 \%)$ & $44(100 \%)$ & $76(100 \%)$ \\
\hline
\end{tabular}

Figures in parenthesis indicate percentage $\mathrm{X}^{2}-0.733 \mathrm{P}>0.05$ 
Thrombocytopenia was noted in 8 out of 23 cases of mild PIH. It was observed that 16 cases of pre-eclampsia had associated thrombocytopenia and 8 cases of eclampsia had thrombocytopenia, which was not statically significant $(\mathrm{P}>0.05)$.

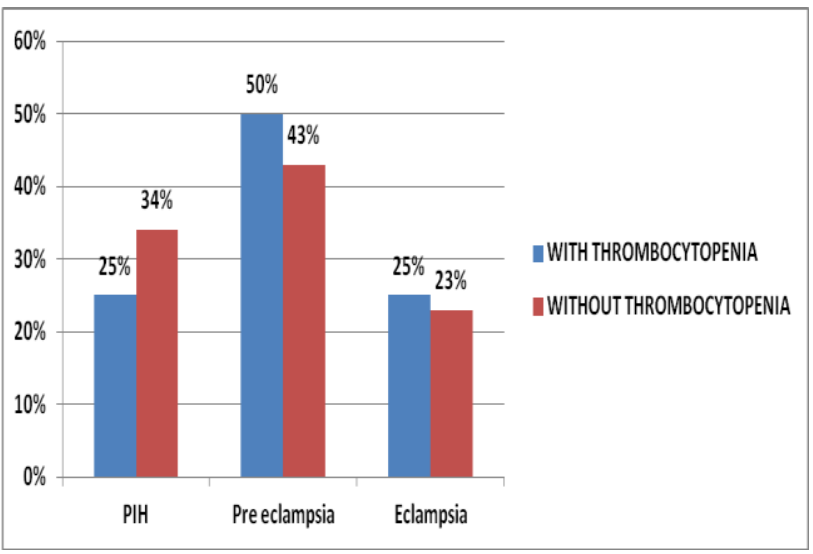

Figure 1: Analysis of severity of pregnancy induced hypertension with and without thrombocytopenia.

Table 4: Analysis of gestational age in pregnancy induced hypertension with and without thrombocytopenia.

\begin{tabular}{|llll|}
\hline \multirow{2}{*}{$\begin{array}{l}\text { Gestational } \\
\text { age }\end{array}$} & \multicolumn{2}{l}{ Thrombocytopenia } & Total \\
\cline { 2 - 4 } With & Without & \\
\hline $35-34 \mathrm{wk}$ & $15(46)$ & $22(68)$ & $37(49 \%)$ \\
\hline$>37 \mathrm{Wk}$ & $10(31)$ & $13(40)$ & $23(30 \%)$ \\
\hline Total & $7(21)$ & $9(27)$ & $16(21 \%)$ \\
\hline
\end{tabular}

Figures in parenthesis indicate percentage

In our study it is observed that maximum cases were between 28 to 34 weeks that is 37 cases out of these 15 cases had thrombocytopenia. In the gestational age of 35 to 37 weeks there were 10 cases associated with thrombocytopenia out of $23(30 \%)$ cases.

In the gestational age of more than 37 weeks 7 has thrombocytopenia out of 16 cases.

Table 5: Distribution of sample according to severity of pregnancy induced hypertension with and without thrombocytopenia in the gestational age group of 28 to 34 weeks.

\begin{tabular}{|c|c|c|c|}
\hline & \multicolumn{2}{|c|}{ Thrombocytopenia } & \multirow{2}{*}{ Total } \\
\hline & With & Without & \\
\hline $\mathrm{PIH}$ & $2(13)$ & $5(23)$ & $7(9)$ \\
\hline Pre eclampsia & $8(53)$ & $11(50)$ & $19(25)$ \\
\hline Eclampsia & $5(33)$ & $6(27)$ & $11(14)$ \\
\hline Total & $15(100 \%)$ & $22(100 \%)$ & $37(100 \%)$ \\
\hline
\end{tabular}

Figures in parenthesis indicate percentage

$\mathrm{X}^{2}=0.546 \mathrm{P}>0.05$
In the gestational age of 28 to 34 weeks, there have been 37 cases of pregnancy induced hypertension out of which 15 cases had thrombocytopenia.

Out of 7 cases of mild pregnancy induced hypertension 2 had thrombocytopenia and 5 did not have thrombocytopenia. In the 19 cases of pre eclampsia it has been noticed that 8 had thrombocytopenia. And the remaining 11 did not have thrombocytopenia. There are 5 cases with thrombocytopenia in the 11 cases of eclampsia and 6 cases without thrombocytopenia.

No statically significant association noted $(\mathrm{P}>0.05)$.

Table 6: Analysis of mode of delivery in patients with or without thrombocytopenia in the gestational age group of 28 to 34 weeks.

\begin{tabular}{|c|c|c|c|}
\hline & \multicolumn{2}{|c|}{ Thrombocytopenia } & \multirow{2}{*}{ Total } \\
\hline & With & Without & \\
\hline VD & $7(13)$ & $10(23)$ & $17(45 \%)$ \\
\hline ID & $2(53)$ & $5(50)$ & $7(18 \%)$ \\
\hline LSCS & $6(33)$ & $7(27)$ & $13(35 \%)$ \\
\hline Total & $15(100 \%)$ & $22(100 \%)$ & $37(100 \%)$ \\
\hline
\end{tabular}

Figures in parenthesis indicate percentage $\mathrm{X}^{2}=0.589 \mathrm{P}>0.05$

Out of the 37 cases of pregnancy induced hypertension in the gestational age of 28 to 34 weeks, 17 (45\%) had vaginal delivery, $7(18 \%)$ had instrumental delivery and $13(35.1 \%)$ underwent cesearen delivery.

The presence or absence of thrombocytopenia had no significant association in deciding the mode of delivery in the gestational age group of 28 to 3 weeks $(\mathrm{P}>0.05)$.

Table 7: Distribution of sample according to severity of pregnancy induced hypertension with and without thrombocytopenia in the gestational age group of $\mathbf{3 5}$ to 37 weeks.

\begin{tabular}{|llll|}
\hline \multicolumn{1}{c}{ Whrombocytopenia } & Total \\
\hline PIH & $3(30)$ & $5(38)$ & $8(34)$ \\
\hline Pre eclampsia & $5(50)$ & $5(38)$ & $10(43)$ \\
\hline Eclampsia & $2(20)$ & $3(23)$ & $5(21)$ \\
\hline Total & $10(100 \%)$ & $13(100 \%)$ & $23(100 \%)$ \\
\hline
\end{tabular}

Figures in parenthesis indicate percentage $\mathrm{X}^{2}=0.314 \mathrm{P}>0.05$

In the gestational age of 35 to 37 weeks, there have been 23 cases of pregnancy induced hypertension out of which 10 cases had thrombocytopenia.

Out of 8 cases of mild pregnancy induced hypertension 3 had thrombocytopenia and 5 did not have thrombocytopenia. In the 10 cases of pre eclampsia it has been noticed that 5 had thrombocytopenia. And the remaining 5 did not have thrombocytopenia. There are 2 cases with thrombocytopenia in the 5 cases of eclampsia.

No statically significant association noted $(\mathrm{P}>0.05)$. 
Table 8: Analysis of mode of delivery in patients with or without thrombocytopenia in the gestational age group of 35 to 37 weeks.

\begin{tabular}{|c|c|c|c|}
\hline & \multicolumn{2}{|c|}{ Thrombocytopenia } & \multirow{2}{*}{ Total } \\
\hline & With & Without & \\
\hline VD & $2(13)$ & $8(23)$ & $10(43 \%)$ \\
\hline ID & $3(53)$ & $2(50)$ & $5(22 \%)$ \\
\hline LSCS & $5(33)$ & $3(27)$ & $8(35 \%)$ \\
\hline Total & $10(100 \%)$ & $13(100 \%)$ & $23(100 \%)$ \\
\hline
\end{tabular}

Figures in parenthesis indicate percentage

$\mathrm{X}^{2}=3.976, \mathrm{P}>0.05$

Out of the 23 cases of pregnancy induced hypertension in the gestational age of 35 to 37 weeks, 10 (43\%) had vaginal delivery, $5(22 \%)$ had instrumental delivery and 8 $(35 \%)$ underwent cesearen delivery.

The presence or absence of thrombocytopenia had no significant association in deciding the mode of delivery in the gestational age group of 35 to 37 weeks $(\mathrm{P}>0.05)$.

Table 9: Distribution of sample according to severity of pregnancy induced hypertension with and without thrombocytopenia in the gestational age group of above 37 weeks.

\begin{tabular}{|c|c|c|c|}
\hline & \multicolumn{2}{|c|}{ Thrombocytopenia } & \multirow{2}{*}{ Total } \\
\hline & With & Without & \\
\hline $\mathrm{PIH}$ & $3(43)$ & $5(56)$ & $8(50)$ \\
\hline Pre eclampsia & $3(43)$ & $5(33)$ & $6(37)$ \\
\hline Eclampsia & $1(14)$ & $1(11)$ & $2(12)$ \\
\hline Total & $7(100 \%)$ & $9(100 \%)$ & $16(100 \%)$ \\
\hline
\end{tabular}

Figures in parenthesis indicate percentage

$\mathrm{X}^{2}=0.254 \mathrm{P}>0.05$

In the gestational age of above 37 weeks, there have been 16 cases of pregnancy induced hypertension out of which 7 cases had thrombocytopenia.

Out of 8 cases of mild pregnancy induced hypertension 3 had thrombocytopenia and 5 did not have thrombocytopenia. In the 6 cases of pre eclampsia it has been noticed that 3 had thrombocytopenia. And the remaining 5 did not have thrombocytopenia. There was 1 case with thrombocytopenia in the 2 cases of eclampsia.

No statically significant association noted $(\mathrm{P}>0.05)$.

Table 10: Analysis of mode of delivery in patients with or without thrombocytopenia in the gestational age group of above 37 weeks.

\begin{tabular}{|llll|} 
& \multicolumn{3}{l}{ Thrombocytopenia } \\
& With & Without & Total \\
\hline VD & $2(13)$ & $4(44)$ & $6(37 \%)$ \\
\hline ID & - & $2(22)$ & $2(12 \%)$ \\
\hline LSCS & $5(71)$ & $3(33)$ & $8(50 \%)$ \\
\hline Total & $7(100 \%)$ & $9(100 \%)$ & $16(100 \%)$ \\
\hline
\end{tabular}

Figures in parenthesis indicate percentage

$\mathrm{X}^{2}=2.963, \mathrm{P}>0.05$
Out of the 7 cases of thrombocytopenia in the gestational age of above 37 weeks, 2 (13\%) had vaginal delivery and $5(71 \%)$ underwent cesearen delivery.

An increased incidence of cesarean delivery was noted in cases with thrombocytopenia in the gestational age of above 37 weeks, probably done in view of better fetal outcome and better survival of the baby but it is not statistically significant $(\mathrm{P}>0.05)$.

Table 11: Analysis of liver enzyme estimation of thrombocytopenia.

\begin{tabular}{|c|c|c|c|}
\hline \multirow{2}{*}{ Liver enzymes } & \multicolumn{2}{|c|}{ Thrombocytopenia } & \multirow{2}{*}{ Total } \\
\hline & With & Without & \\
\hline Elevated & $18(56)$ & $17(39)$ & $35(46 \%)$ \\
\hline Normal & $14(44)$ & $27(61)$ & $41(53 \%)$ \\
\hline Total & $32(100 \%)$ & $44(100 \%)$ & $76(100 \%)$ \\
\hline
\end{tabular}

Figures in parenthesis indicate percentage

$\mathrm{X}^{2}=2.313, \mathrm{P}>0.05$

There has been no statically significant association noted between liver enzymes and thrombocytopenia.

Table 12: Distribution of subjects according to classification of HELLP.

\begin{tabular}{|ll|}
\hline HELLP & Total \\
\hline Class I & $1(13 \%)$ \\
\hline Class II & $3(38 \%)$ \\
\hline Class III & $4(50 \%)$ \\
\hline Total & $8(10.5 \%)$ \\
\hline
\end{tabular}

In our study, HELLP syndrome was classified according to Mississippi classification ie based upon the platelet count. Class III constituted the maximum percentage of cases followed by Class II. Class I had the least number of cases.

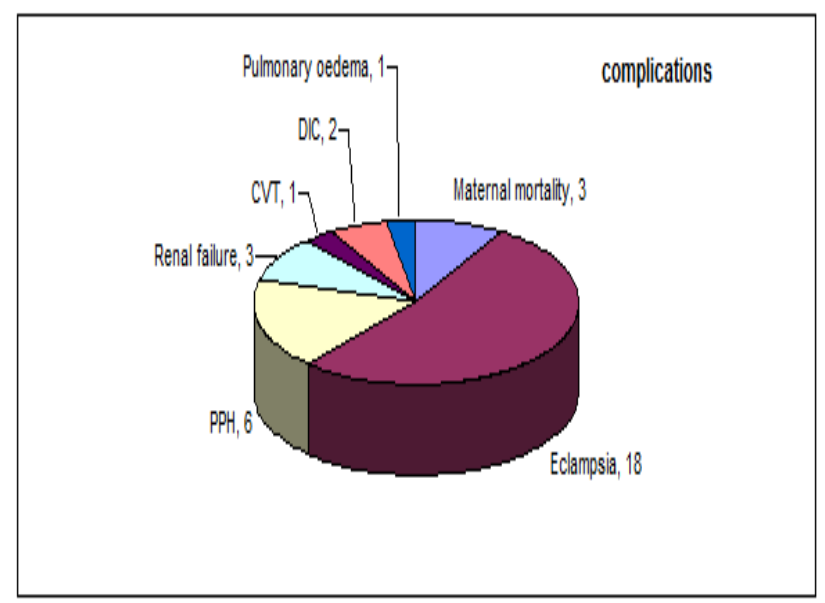

Figure 2: Maternal complications. 
In our study, maternal outcome was thoroughly analysed and the above complications were noted. Out of the 76 cases, there were 3 cases of maternal mortality due to PPH, eclampsia and abruption leading to DIC.

Table 13: Maternal complications.

\begin{tabular}{|ll|}
\hline HELLP & Total \\
\hline Maternal mortality & 3 \\
\hline Eclampsia & 18 \\
\hline PPH & 6 \\
\hline Renal failure & 3 \\
\hline CVT & 1 \\
\hline DIC & 2 \\
\hline Pulmonary oedema & 1 \\
\hline
\end{tabular}

Table 14: Analysis of maternal outcome with or without thrombocytopenia.

\begin{tabular}{|llll|}
\hline Maternal & \multicolumn{2}{l}{ Thrombocytopenia } & Total \\
outcome & With & Without & \\
\hline Improved & 18 & 42 & $60(87 \%)$ \\
\hline Morbidity & 11 & 2 & $13(9 \%)$ \\
\hline Mortality & 3 & 0 & $3(4 \%)$ \\
\hline Total & $32(100 \%)$ & $44(100 \%)$ & $76(100 \%)$ \\
\hline
\end{tabular}

Figures in parenthesis indicate percentage

In our study, there were 3 cases of maternal mortality due to PPH, eclampsia which deteriorated to cerebral oedema $\&$ cerebral damage leading to death and abruption leading to DIC.

The percentage of morbidity was $9 \%$, the causes of which were diverse and included PPH, DIC, abruption, renal failure, multiorgan failure, pulmonary oedema and CVT.

It has definitely been noted that the earlier and more aggressive treatment of pregnancy induced hypertension has resulted in better maternal outcome.

Table 15: Analysis of fetal outcome in pregnancy induced hypertension with and without thrombocytopenia.

\begin{tabular}{|llll|}
\hline Fetal & \multicolumn{2}{l}{ Thrombocytopenia } & Total \\
\cline { 2 - 3 } outcome & With & Without & \\
\hline Healthy & 10 & 24 & $34(44 \%)$ \\
\hline $\begin{array}{l}\text { Perinatal } \\
\text { morbidity }\end{array}$ & 12 & 11 & $23(30 \%)$ \\
\hline $\begin{array}{l}\text { Perinatal } \\
\text { mortality }\end{array}$ & 10 & 9 & $19(25 \%)$ \\
\hline Total & $32(100 \%)$ & $44(100 \%)$ & $76(100 \%)$ \\
\hline
\end{tabular}

Figures in parenthesis indicate percentage

Fetal outcome is influenced by gestational age and mode of delivery. In our study, there is a perinatal outcome in the group without thrombocytopenia but it is not statistically significant.

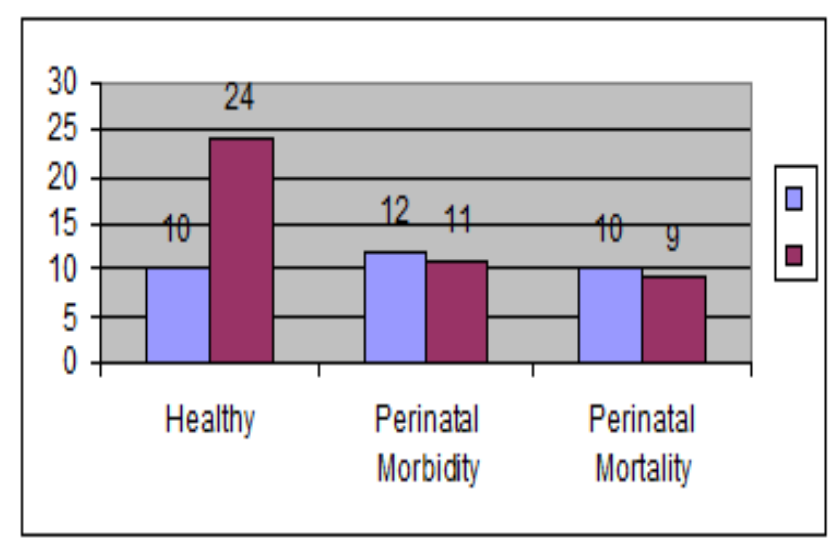

Figure 3: Analysis of fetal outcome in pregnancy induced hypertension with and without thrombocytopenia.

\section{DISCUSSION}

Pre eclampsia affects approximately $6 \%$ of all pregnancies. Women with severe pre-eclampsia may develop variety of hematological aberrations. Thrombocytopenia complicating pregnancy is reported relatively frequently in severe pre eclampsia with occurrence ranging at 11 to $29 \% .^{22,23}$ The incidence of thrombocytopenia associated with pregnancy induced hypertension in our study was $42.11 \%$, which is much higher than earlier reported cases.

This high incidence may be explained due to hospital admission of only those patients who are willing for admission and are ill enough to seek for hospital care. The use of thrombocytopenia as a screening test for severity of pregnancy induced hypertension has not been well documented in the literature. Redman et al reported decreased platelet count in women who developed preeclampsia and stated that increased platelet consumption is an early feature of this disorder. ${ }^{24}$

Galton et al claimed that the severity of thrombocytopenia correlates with severity of hypertension. $^{25}$

Romero et al reported that women with pre eclampsia and thrombocytopenia have higher incidence of maternal and neonatal complications. ${ }^{22}$

The patients in our study are well defined group with diagnosed hypertensive disorder of pregnancy. All patients have been accurately randomized using the inclusion and exclusion criteria. Complete hemogram including platelet count has been done and documented. The cases have been followed up for maternal and perinatal outcome. In our study, out of 76 cases of hypertensive disorders of pregnancy 32 cases had documented thrombocytopenia which accounts for $42.11 \%$. 
There has been no statistically significant association between the severities of pregnancy induced hypertension and thrombocytopenia. But a higher incidence of cesarean section was observed with increasing gestational age, probably because of better fetal outcome and fetal survivability. There is no statistically significant association between maternal outcome and thrombocytopenia but in cases with severe thrombocytopenia, there is an increase incidence of maternal morbidity due to abruption placenta, DIC, renal failure, CVT, eclampsia and pulmonary oedema. There were 3 cases of maternal mortality due to uncontrolled post-partum haemorrhage, eclampsia and abruption placenta leading to DIC. The perinatal outcome mainly depended upon the gestational age at the time of delivery. Better fetal outcomes were reported with increasing gestational age. The previous studies showed a poorer fetal outcome but our study reports better fetal outcome due to prompt administration of steroids and better management of neonatal care.

\section{CONCLUSIONS}

Thrombocytopenia in hypertensive disorders of pregnancy carries a definite risk to the mother and fetus. There is a relation between thrombocytopenia and associated complications of pregnancy induced hypertension like abruptio, intrauterine fetal demise, septicemia and DIC. This is seen especially more in early onset hypertension and carries severe risk of morbidity and mortality to mother and fetus. Another dreaded complication of severe hypertension is HELLP syndrome, which causes a dilemma for obstetric health care providers because of nonspecific signs and symptoms early in the disease process which delays accurate diagnosis and early treatment. In cases of HELLP syndrome which receive early treatment, there has been good prognosis in terms of maternal and fetal outcome. Thrombocytopenia did not affect the mode of delivery.

It is observed that mild thrombocytopenia is common in third trimester and most often has a benign course.

Prematurity has been the most common complication for the fetus and timely administration of corticosteroid will enhance the lung maturity and reduce the risk of intraventricular hemorrhage and necrotizing enterocolitis between 28 to 34 wks, thereby reducing the perinatal mortality.

In our study platelet count of less than 1, 50,000 was shown to have borderline utility in predicting the risk of adverse maternal outcome. This may be due to some undiagnosed bias of randomization of study population or loss of samples due to referrals and basically a smaller study group. In the end it can be safely concluded that early diagnosis and appropriate early treatment of pregnancy induced hypertension results in better maternal and fetal outcome ensuring a good health care system in the population.

Funding: No funding sources

Conflict of interest: None declared

Ethical approval: The study was approved by the Institutional Ethics Committee

\section{REFERENCES}

1. Mohapatra S, Pradhan BB, Satpathy UK, Mohanty A, Pattnaik JR. Platelet estimation: Its prognostic value in pregnancy induced hypertension. Indian $\mathrm{J}$ Physiol Pharmacol. 2007;51(2):160-4.

2. McCrae KR. Pregnancy induced thrombocytopenia: pathogenesis and management. Blood. 1992;80;2697-714.

3. Charo IF, Kieffer N. Platelet membrane glycoproteins. Hemostasis and thrombin; Basic principles and clinical practice, Philadelphia: JB Lippincott co. 1994;489-507.

4. Lee GR, Fester J. Thrombocytopenia pathophysiology and classification. Wintrobe's clinical hematology. 10th edition. Philadelphia: lippincott Williams \& Wilkins. 1999:1579-1611.

5. Rodgers RPC, Levin J. A critical reappraisal of bleeding time. Sem throm hemost. 1990;16:1-144.

6. Kumar V, Abbas AK. Pathologic basis of disease. Diseases of immune system. 8th edition. 2007;5:11134.

7. Branchog I. Platelet enemal and platelet production in ITP. British Journal of Hematology. 1974;27:12743.

8. Harker LD. Thrombo kinelin in man. J. Clin invest. 1969;48:963-74.

9. Meaell JS. Antenatal management of thrombocytopenias. Clin perinatal. 1994;21:591-614.

10. Greer IA. A prospective longitudinal study of immuno reactive prostacyclin and TX-A2 metabolites in normotensive and hypertensive pregnancy. Clinical and experimental hypertension. Part B: hypertension in pregnancy. 1985;4:167-81.

11. Gowland E. Agglutination of platelets by a serum factor in the presence of EDTA. J. Clinicopathol. 1969;22:460-64.

12. Kield, Berg CR. Spurious thrombocytopenia. JAMA. 1974;227:628-30.

13. Burrows RF. Fetal thrombocytopenia and its relationship to maternal thrombocytopenia. N. Engl J Med. 1993;329:1463-6.

14. Burrows RF. Low fetal risks in pregnancies associated with idiopathic thrombocytopenic purpura. Am J Obstetrics \& Gynecol. 1990;163:1147-50.

15. Kenton JG. The serological investigation of patients with autoimmune thrombocytopenia. Thromb hoemost. 1995; 74:228-33.

16. Berchtold. Autoantibodies against platelet glycoproteins in autoimmune thrombocytopenic 
purpura, their clinical significance and response to treatment. Blood. 1993;81:1246-50.

17. Kruayenbrink AA. Endothelial vasoactive mediation in pre eclampsia. Am J Obstet Gynecol. 1993;169:160-3.

18. Barton JR. Care of pregnancy complicated by HELLP syndrome. Clin N Am. 1991;18:165-79.

19. Audibert F. Clinical utility of strict diagnostic criteria for HELLP syndrome. Am J Obst Gynecol. 1996;175:460-64.

20. Sibai BM. Pregnancies complicated by HELLP syndrome, subsequent pregnancy outcomes, long term prognosis. Am J Obst Gynecol. 1995;172:1259.

21. Visser W. Management of severe pre eclampsia with and without HELLP syndrome. Br J obstet \& gynecol. 1995;102:111-7.
22. Romero R. Clinical significance, prevalence and natural history of thrombocytopenia in pregnancy induced hypertension. Am J perinatal. 1989;6:32-8.

23. Prichart JA. Coagulation changes in eclampsia: their frequency and pathogenesis. Am J Obstet Gynecol. 1976;4:855-64

24. Redman CW. Early platelet consumption in preeclampsia. Br J med. 1978;70:334-8.

25. Galton M. Coagulation studies on the peripheral circulation of patients with toxemia of pregnancy. J Reprod med. 1971;6:89-100.

Cite this article as: Tejeswini KK, Anitha GS, Nandagopal KM. Platelet count as a prognostic indicator in pregnancy induced hypertension. Int $\mathbf{J}$ Reprod Contracept Obstet Gynecol 2016;5:1036-46. 